\title{
Rivaroxaban Attenuates Right Ventricular Remodeling in Rats with Pulmonary Arterial Hypertension
}

\author{
Hideki Imano, ${ }^{a}$ Ryuji Kato, ${ }^{a}$ Atsuo Nomura,,${ }^{a, b}$ Maki Tamura, ${ }^{a}$ Yudai Yamaguchi, ${ }^{a}$ Yoshio Ijiri, ${ }^{a}$ \\ Hong Wu, ${ }^{c}$ Takashi Nakano, ${ }^{c}$ Yoshikatsu Okada, ${ }^{d}$ Takehiro Yamaguchi, ${ }^{e}$ Yasukatsu Izumi, ${ }^{e}$ \\ Minoru Yoshiyama, ${ }^{f}$ Michio Asahi, ${ }^{b}$ and Tetsuya Hayashi*, ${ }^{\circ}$ \\ ${ }^{a}$ Department of Cardiovascular Pharmacotherapy and Toxicology, Osaka University of Pharmaceutical Sciences; \\ Takatsuki, Osaka 569-1094, Japan: ${ }^{b}$ Department of Pharmacology, Faculty of Medicine, Osaka Medical College; \\ Takatsuki, Osaka 569-8686, Japan: ' Department of Microbiology and Infection Control, Faculty of Medicine, \\ Osaka Medical College; Takatsuki, Osaka 569-8686, Japan: ${ }^{d}$ Department of Pathology, Faculty of Medicine, \\ Osaka Medical College; Takatsuki, Osaka 569-8686, Japan: ${ }^{e}$ Department of Pharmacology, Osaka City University \\ Graduate School of Medicine; Osaka 545-8585, Japan: and ${ }^{f}$ Department of Cardiovascular Medicine, Osaka City \\ University Graduate School of Medicine; Osaka 545-8585, Japan. \\ Received December 18, 2020; accepted February 11, 2021; advance publication released online February 20, 2021
}

Pulmonary arterial hypertension (PAH) is a progressive condition that frequently results in right ventricular (RV) remodeling. The objectives of this study are to investigate effects of rivaroxaban on $R V$ remodeling in a rat model of $\mathrm{PAH}$, created with Sugen5416 and chronic hypoxia, and the in vitro effects of rivaroxaban on human cardiac microvascular endothelial cells (HCMECs). To create the PAH model, male Sprague-Dawley rats were subcutaneously injected with Sugen5416 (20 mg/kg) and exposed to 2 weeks of hypoxia $\left(10 \% \mathrm{O}_{2}\right)$, followed by 2 weeks of exposure to normoxia. The animals were then divided into 2 groups with or without administration of rivaroxaban $(12 \mathrm{mg} / \mathrm{kg} / \mathrm{d})$ for a further 4 weeks. HCMECs were cultured under hypoxic conditions $\left(37^{\circ} \mathrm{C}, 1 \% \mathrm{O}_{2}, 5 \% \mathrm{CO}_{2}\right)$ with Sugen5416 and with or without rivaroxaban. In the model rats, RV systolic pressure and Fulton index increased by hypoxia with Sugen5416 were significantly decreased when treated with rivaroxaban. In HCMECs, hypoxia with Sugen5416 increased the expression of protease-activated receptor-2 (PAR-2) and the phosphorylation of extracellular signal-regulated kinase (ERK), c-Jun N-terminal kinase (JNK), and nuclear factor-kappa B (NF- $\kappa$ B), while treatment with rivaroxaban significantly suppressed the expression of these proteins. Rivaroxaban attenuated RV remodeling in a rat model of PAH by reducing ERK, JNK and NF- $\kappa$ B activation. Rivaroxaban has the possibility of providing additive effects on $\mathrm{RV}$ remodeling in patients with PAH.

Key words rivaroxaban; Sugen5416; right ventricular remodeling; pulmonary arterial hypertension

\section{INTRODUCTION}

Pulmonary arterial hypertension (PAH) is a progressive condition that frequently results in right ventricular (RV) remodeling and heart failure. ${ }^{1)}$ Currently, there is no curative treatment for PAH except lung transplantation. To investigate the pathogenic mechanisms of this condition, a rat model has been developed with similar histological features to those of patients with PAH. ${ }^{2)}$

Sugen5416 (semaxinib) is a multi-kinase inhibitor that blocks angiogenesis through the inhibition of the vascular endothelial growth factor receptor. ${ }^{3)}$ In our previous study, the earliest pathological change observed in the rat model of PAH induced by Sugen5416 administration and exposure to sustained hypoxia (PAH model rats) was the degeneration of capillaries in the heart that resulted in accelerated RV remodeling. ${ }^{4)}$

Thrombus formation is commonly associated with PAH. One of the recommendations for treating PAH given in the practice guidelines by the American College of Chest Physicians, is to use anticoagulation therapy with warfarin. ${ }^{5)}$ However, a therapeutic benefit of anticoagulation has not yet been clearly demonstrated.

Rivaroxaban is an oral direct inhibitor of coagulation factor $\mathrm{X}(\mathrm{FXa})$ and the prothrombinase complex. $\left.{ }^{6}\right)$ In an experimen- tal model of PAH, Delbeck et al. demonstrated that rivaroxaban displays dose-dependent anticoagulant activity and is more effective than warfarin. ${ }^{7)}$ Protease-activated receptor-2 (PAR-2) is upregulated both in idiopathic human PAH and in animal models of pulmonary hypertension, while inhibition of PAR-2 reverses experimental pulmonary hypertension induced by hypoxia. ${ }^{8)}$ Rivaroxaban significantly reduces oxidative stress and myocardial fibrosis via inhibition of PAR-2, and attenuates cardiomyocyte hypertrophy and capillary degeneration caused by intermittent hypoxia. ${ }^{9)}$ These findings suggest that rivaroxaban could have a preventive effect on cardiac remodeling induced by hypoxia.

As outlined above, rivaroxaban is potentially useful for treating PAH, and could be beneficial for PAH patients. However, little is reported regarding the effects of rivaroxaban on the heart in PAH models and the mechanisms involved. This prompted us to investigate the effects of rivaroxaban on right ventricular remodeling by using a rat model of PAH.

\section{MATERIALS AND METHODS}

Experimental Protocol Seven-week-old Sprague-Dawley rats $(n=36)$ were purchased from Japan SLC Inc. (Hamamatsu, Shizuoka, Japan), and were maintained in an animal room under a 12-h light-dark cycle with free access to standard 
laboratory chow and tap water. The rats underwent subcutaneous injection of Sugen5416 (20 mg/kg; Merck KGaA, Darmstadt, Germany) and then were exposed to 2 weeks of hypoxia $\left(10 \pm 1 \% \mathrm{O}_{2}\right)$, followed by 2 weeks of exposure to normoxia. The rats were then divided into 2 groups and received either no treatment (SuHx group) or rivaroxaban $(12 \mathrm{mg} / \mathrm{kg} / \mathrm{d}$, SuHx + RIV group) for 4 weeks. Rivaroxaban was supplied by Bayer Pharma AG, Wuppertal, Germany. Rats maintained under normoxic conditions in the same room served as controls (Normoxia group). On the day after completion of the 4-week period, cardiac catheterization was performed to assess cardiac function. The heart was then excised in order to dissect the RV from the left ventricle and septum.

The experimental protocol and animal handling procedures for this study were reviewed and approved by the Experimental Animal Research Committee of Osaka University of Pharmaceutical Sciences.

Cell Culture Human cardiac microvascular endothelial cells (HCMECs; LONZA Japan, Tokyo, Japan) were cultured in tissue culture flasks of $25 \mathrm{~cm}^{2}$ (Becton-Dickinson Ind., $\mathrm{NJ}$, U.S.A.), and then maintained at $37^{\circ} \mathrm{C}$ in $5 \% \mathrm{CO}_{2}$ using the $\mathrm{EGM}^{\mathrm{TM}}-2 \mathrm{MV}$ BulletKit ${ }^{\mathrm{TM}}$ (LONZA Japan). Cells were treated with $0.25 \%$ trypsin $/ 1 \mathrm{mM}$ ethylenediaminetetraacetic acid (EDTA) in $\mathrm{Ca}^{2+}$ - and $\mathrm{Mg}^{2+}$-free phosphate-buffered saline (PBS) and subcultured every $7 \mathrm{~d}$. Following trypsin digestion and centrifugation, the cells were resuspended in $5 \mathrm{~mL}$ of medium at a concentration of $5 \times 10^{5}$ cells $/ \mathrm{mL}$. Cell growth and contamination were monitored regularly by examining the cultures under an inverted light microscope. Cells were incubated $\left(37^{\circ} \mathrm{C}, 24 \mathrm{~h}\right)$ in either a $5 \% \mathrm{CO}_{2}$ and $20 \% \mathrm{O}_{2}$ environment (Normoxia group) or in $5 \% \mathrm{CO}_{2}$ and $1 \% \mathrm{O}_{2}$ environment with Sugen5416 $(3 \mu \mathrm{M})$. Then the culture medium was aspirated and cells were washed by PBS. Culture medium with dimethyl sulfoxide (SuHx group) or rivaroxaban $(\mathrm{SuHx}+\mathrm{RIV}$ group) was added and the cells were incubated $\left(37^{\circ} \mathrm{C}\right)$ for another $24 \mathrm{~h}$ in $5 \% \mathrm{CO}_{2}$. The concentration of $1 \mu \mathrm{M}$ of rivaroxaban was used.

Cardiac Catheterization The rats were anesthetized using pentobarbital sodium $(50 \mathrm{mg} / \mathrm{kg})$ after being treated with either Sugen 5416 or vehicle. RV pressures were measured by inserting a 1.4-Fr Micro-tip catheter (model SPR-671, Millar Instruments, Houston, TX, U.S.A.) into the right jugular vein or the right carotid artery.

Echocardiography Interventricular septum (IVS), left ventricular (LV) posterior wall (PW) thickness, LV fractional shortening (FS), and RV fractional area change (FAC) were obtained through M-mode transthoracic echocardiography performed with a Vivid E9 instrument (GE Healthcare, Salt Lake City, UT, U.S.A.). RV global systolic function was assessed as the tricuspid annular plane systolic excursion (TAPSE), which reflects basoapical systolic RV shortening. ${ }^{10-12)}$ An index of global systolic and diastolic ventricular function (RV Tei index) was calculated by subtracting the ejection time from the sum of the isovolumic contraction time and the isovolumic relaxation time and then dividing this result by the ejection time.

Histological Assessment In preparation for the light microscopy, cardiac and pulmonary tissues were fixed in $10 \%$ formaldehyde and embedded in paraffin. These tissues were then cut into $4-\mu \mathrm{m}$ sections, and with an ECLIPSE 80i light microscope (Nikon, Tokyo, Japan), photomicrographs were obtained.

The cardiac cross-sectional area was measured from the photomicrographs using version 1.44 of the ImageJ software (National Institutes of Health, MD, U.S.A.) at a magnification of $400 \times$ by a method described in a previous paper. ${ }^{13)}$

The Fulton index was calculated from dividing the weight of RV by the total weight of the septum and the LV. In each heart, at least 30 cardiomyocytes with a clearly visible nucleus and intact cell membrane were examined using hematoxylineosin-stained nucleated transverse sections.

The collagen volume ratio (\%) was calculated after staining with Sirius red [1].

In preparation for the electron microscopy, the RV myocardium was fixed in $4 \%$ paraformaldehyde containing $0.25 \%$ glutaraldehyde and $4.5 \%$ sucrose. Ultrathin sections were then cut and stained with uranyl acetate and lead citrate from the fixed tissues. These were examined using a Hitachi H-7650 model electron microscope (Hitachi, Tokyo, Japan). ${ }^{14)}$

Immunohistochemistry for 4-Hydroxy-2-nonenal Immunohistochemical staining was done on paraffin sections of the RV in order to detect 4-hydroxy-2-nonenal (4-HNE)-modified protein adducts. Sections were incubated with a monoclonal antibody for 4-HNE (MHN-100P; Japan Institute for the Control of Aging, Shizuoka, Japan) in 1:80 dilution and with secondary antibodies using Histofine Simple Stain MAX-PO (MULTI) (Nichirei Biosciences, Tokyo, Japan). The area of 4-HNE staining was then compared with the mean area on control sections (defined as 1.0).

Terminal Deoxynucleotidyl Transferase-Mediated Deoxyuridine Triphosphate-Biotin Nick End Labelling (TUNEL) Assay RV myocardial tissue samples were evaluated by the TUNEL method using an Apop Tag peroxidase in situ apoptosis detection kit (Millipore Japan, Tokyo, Japan). Samples incubated with distilled water were used as negative control while endometrium was used as positive control. Once the samples were counterstained with Mayer's haematoxylin, the percentages of TUNEL-positive nuclei of myocardial cells and interstitial cells among the total number of counted nuclei were calculated using a method described elsewhere. ${ }^{15)}$

Quantitative Real-Time RT-PCR TRIzol reagent (Molecular Research Center, OH, U.S.A.) was used to extract total RNA from RV myocardial tissue samples. cDNA was synthesized from the total RNA through reverse transcription using ReverTra Ace qPCR RT Master Mix with cDNA Remover (Toyobo Co., Ltd., Osaka, Japan). The version 2.45 of ProbeFinder was used to design primers and probes for PAR-2, extracellular signal-regulated kinase (ERK)-1, ERK-2, nuclear factor-kappa B (NF- $\kappa$ B), c-Jun N-terminal kinase (JNK), Transforming Growth Factor (TGF)- $\beta$, and 18S ribosomal RNA (rRNA). Using the Roche Universal Probe Library system, this software creates sets of specific primers and selects TaqMan-locked nucleic acid probes. Universal Probes that were purchased from Roche Diagnostics (Basel, Switzerland) are as follows: No. 22 for rat 18S rRNA, No. 63 for rat PAR-2, No. 68 for rat ERK-1, No. 1 for rat ERK-2, No. 77 for rat JNK, No. 1 for rat TGF- $\beta$, and No. 78 for rat NF- $\kappa$ B. The level of mRNA expression was measured using a LightCycler ${ }^{\circledR}$ (Roche Diagnostics) with 18S rRNA as the internal standard. The primer sequences that were used are shown in Table 1.

Western Blotting Protein samples $(20 \mu \mathrm{g})$ were subjected to $12.5 \%$ sodium dodecyl sulfate-polyacrylamide gel 
electrophoresis, followed by electrotransfer of the resolved proteins to a polyvinylidene difluoride membrane $(0.45 \mu \mathrm{m}$, Merck KGaA). The following primary antibodies were used in 1: 1000 dilution: rabbit anti-PAR-2 antibody (bs-1178R, Bioss Antibodies Inc., MA, U.S.A.), rabbit anti-pERK-1/2 antibody (\#9101, Cell Signaling Technology, MA, U.S.A.), rabbit antiERK-1/2 antibody (\#4695, Cell Signaling Technology), rabbit anti-pNF- $\kappa$ B antibody (\#3033, Cell Signaling Technology), rabbit anti-NF- $\kappa$ B antibody (sc-372, Santa Cruz Biotechnology, TX, U.S.A.), rabbit anti-pJNK antibody (\#9251S, Cell Signaling Technology), rabbit anti-JNK antibody (\#9252S, Cell Signaling Technology), rabbit anti-pSmad3 antibody (\#9520S, Cell Signaling Technology), rabbit anti-Smad3 antibody (\#9523S, Cell Signaling Technology), and $\beta$-actin antibody (sc-47778, Santa Cruz Biotechnology). Reaction products were detected with goat anti-rabbit immunoglobulin G

Table 1. Primer Sequences Used for Real-Time RT-PCR Analyses

\begin{tabular}{lll}
\hline \hline Target & Primer forward $5^{\prime} \rightarrow 3^{\prime}$ & Primer reverse $3^{\prime} \rightarrow 5^{\prime}$ \\
\hline PAR-2 & tctcaggaatcccagtgaaga & tcgagcggtatgctttcatt \\
ERK-1 & ccctgtccttttggacctg & gggttgagcaaagttcatttc \\
ERK-2 & tgaagttgaacaggctctgg & tgaatggtgcttcagcaatg \\
JNK & gtatgatccttcagaagcagagg & Catgacctccttgtatatcagttctt \\
NF- $\kappa$ B & tagcaaaccaccaagcagag & ggttgtctttgagatccatgc \\
TGF- $\beta$ & cctggaaagggctcaacac & tgccgtacacagcagttctt \\
18 S rRNA & ggtgcatggccgttctta & tcgttcgttatcggaattaacc \\
\hline
\end{tabular}

a

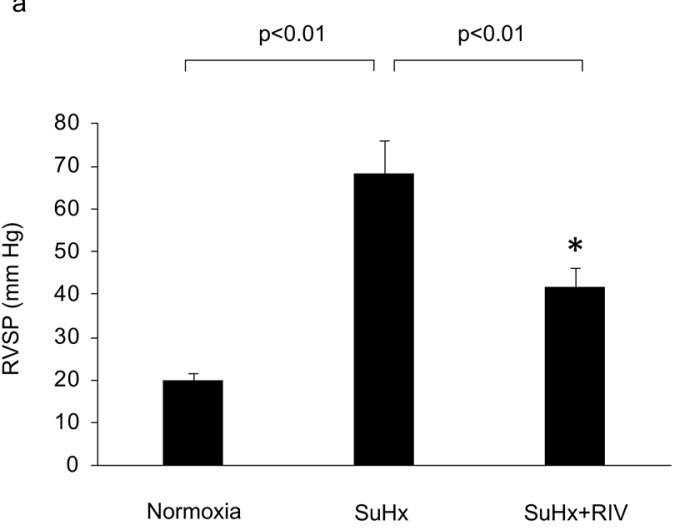

c

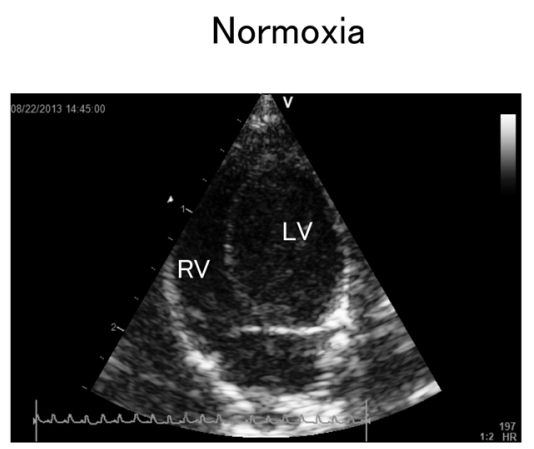

(IgG)-peroxidase (4050-05, Southern Biotech, AL, U.S.A.) in $1: 1000$ dilution, and bound peroxidase was visualized using Luminata $^{\mathrm{TM}}$ Classico Western horseradish peroxidase (Merck $\mathrm{KGaA}$ ). Western blotting was performed three times for each sample.

Statistical Analysis Results are expressed as the mean and standard deviation (S.D.). In order to adjust for multiplicity, the Tukey-Kramer test was performed. $p$-Values less than 0.05 were considered to indicate statistical significance.

\section{RESULTS}

Cardiac Catheterization The cardiac catheterization reveals that RV systolic pressure in the SuHx group was significantly higher than that in the Normoxia group and that RV systolic pressure in the SuHx + RIV group was significantly lower than that in the SuHx group (Fig. 1a).

Echocardiographic Findings Echocardiographic findings showed that ventricular septum and LV posterior wall were thickened in the SuHx group (Table 2). In the apical 4-chamber view, dilated RV and compressed LV with thickened ventricular septum were observed in the SuHx group (Fig. 1c). Treatment with RIV showed little effect on LV systolic function. For the parameters related to RV function, significant differences in the FAC and RV Tei index were found between the SuHx group and the Normoxia group. RV Tei index in the $\mathrm{SuHx}+\mathrm{RIV}$ group was significantly lower than that in the SuHx group (Table 2).

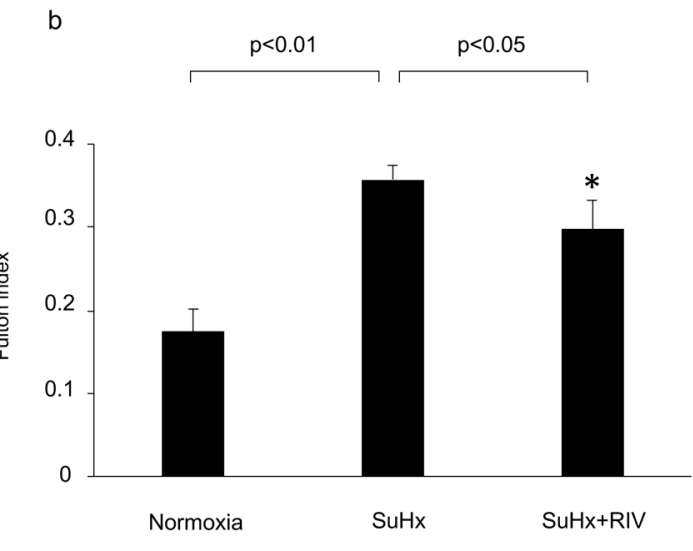


Table 2. Echocardiographic Parameters

\begin{tabular}{lcccccc}
\hline \hline Group & IVS $(\mathrm{mm})$ & PW $(\mathrm{mm})$ & FS $(\%)$ & FAC $(\%)$ & TAPSE $(\mathrm{mm})$ & RV Tei index \\
\hline Normoxia & $1.04 \pm 0.01$ & $1.08 \pm 0.02$ & $39.6 \pm 2.9$ & $42.7 \pm 0.8$ & $2.65 \pm 0.31$ & $0.21 \pm 0.03$ \\
SuHx & $1.61 \pm 0.18^{*}$ & $1.62 \pm 0.18^{*}$ & $46.4 \pm 2.9$ & $31.6 \pm 2.3^{*}$ & $1.45 \pm 0.32$ & $0.66 \pm 0.16^{*}$ \\
SuHx + RIV & $1.42 \pm 0.03^{*}$ & $1.44 \pm 0.08^{*}$ & $43.4 \pm 8.9$ & $35.5 \pm 5.8$ & $2.19 \pm 0.86$ & $0.37 \pm 0.10^{\dagger}$ \\
\hline
\end{tabular}

IVS, interventricular septum; PW, left ventricular posterior wall thickness; FS, fractional shortening; FAC, fractional area change; RV Tei index, right ventricular total ejection isovolume index; LVEF, left ventricular ejection fraction; TAPSE, tricuspid annular plane systolic excursion. Data are shown as the mean and S.D. $(n=3) . * p<0.05, v S$. Normoxia; ${ }^{\dagger} p<0.05$, vs. SuHx.

Normoxia
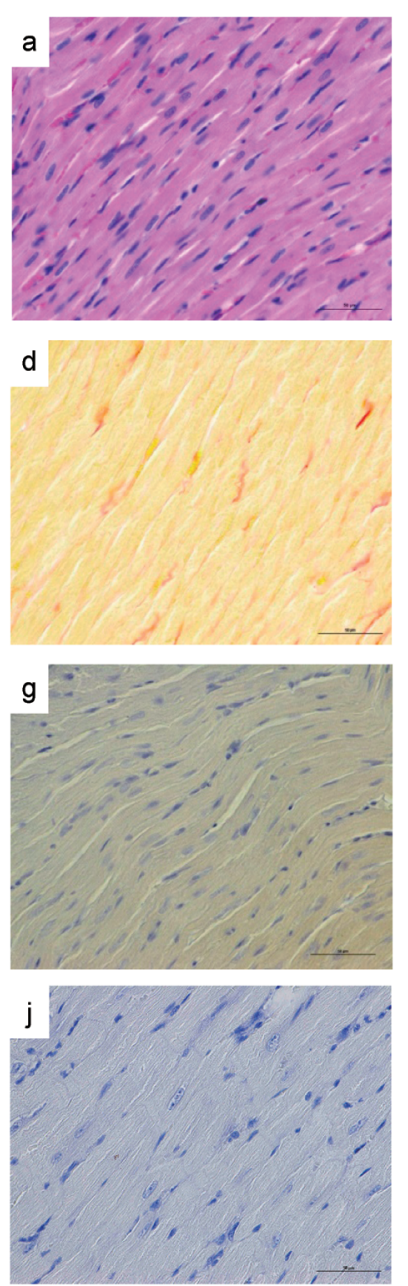

SuHx
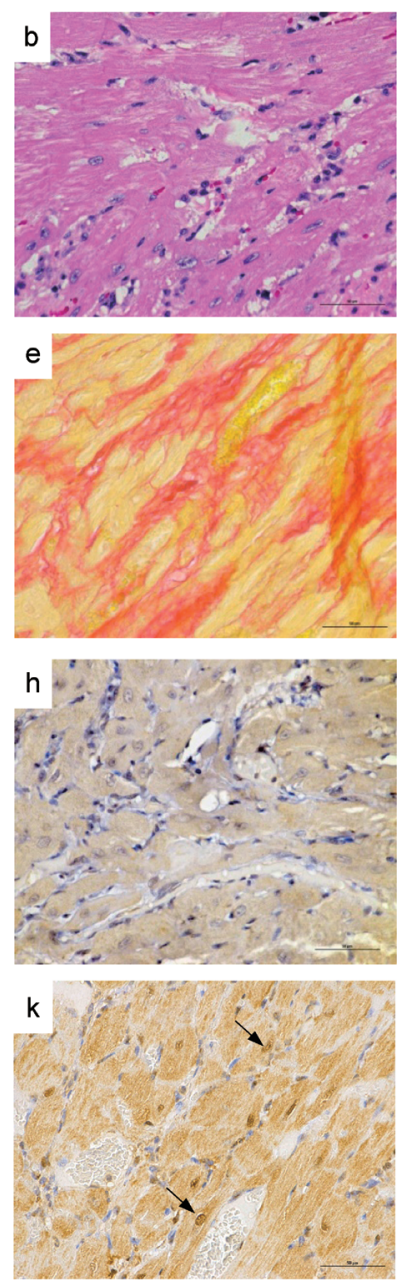

\section{SuHx+RIV}
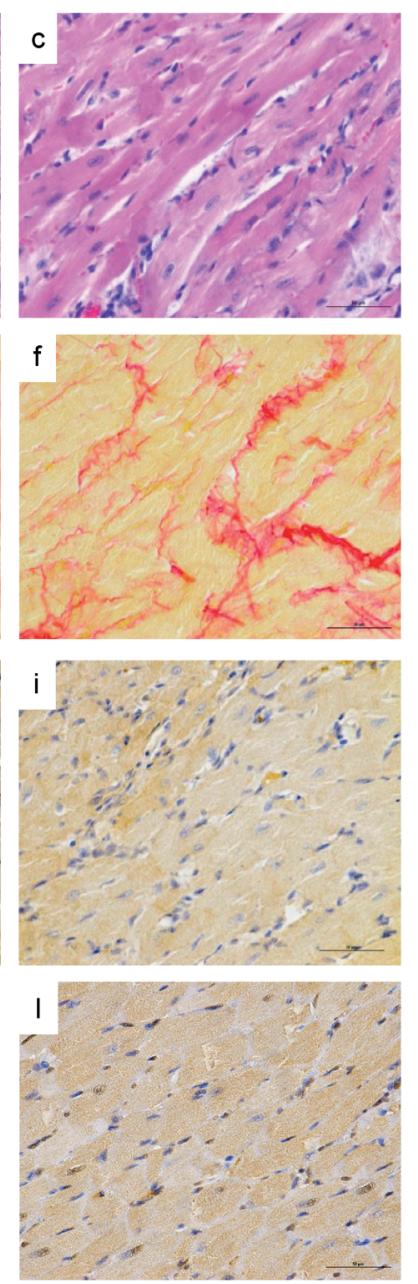

Fig. 2. Hematoxylin-Eosin (H\&E) Staining (a-c), Sirius Red Staining (d-f), Immunohistochemical Staining for 4-HNE-Modified Protein Adducts $(\mathrm{g}-\mathrm{i})$, and Immunohistochemical Staining for TUNEL-Positive Cells $(\mathrm{j}-1)$ of Rat Right Ventricle

In comparison with the Normoxia group ( $\mathrm{a}, \mathrm{d}, \mathrm{g}, \mathrm{j}$ ), subcutaneous injection of Sugen5416 and exposure to hypoxia (SuHx) caused severe degeneration of RV myocardium, including hypertrophy of cardiomyocytes, myofiber disarray and scarcity of myofibers with mononuclear cellular infiltration (b), increased interstitial fibrosis (e), 4-HNE-modified protein adducts (h) and number of TUNEL-positive cells (arrows) (k) in the right ventricular myocardium. Treatment with rivaroxaban led to suppression of these changes (SuHx + RIV) (c, f, i, l).

Histological Findings Fulton index in the SuHx group was significantly increased compared with that in the Normoxia group. In the SuHx + RIV group, Fulton index was significantly decreased compared with that in the SuHx group (Fig. 1b).

Under light microscopy, the RV myocardium displayed severe degeneration of cardiomyocytes, disarray of myofilaments, mononuclear cell infiltration, and interstitial fibrosis in the SuHx group (Figs. 2b, e, Supplementary Fig. 1). In addition, the cardiomyocyte cross-sectional area and interstitial fibrosis were significantly increased. The increase of car- diomyocyte cross-sectional area and interstitial fibrosis was significantly suppressed in the SuHx + RIV group (Figs. 3a, b). Furthermore, increased medial wall thickness in the SuHx group was improved by the treatment with RIV (Supplementary Fig. 2).

Under electron microscopy, ultrastructure of capillaries and RV cardiomyocytes showed normal configuration in the Normoxia group (Fig. 4a). In the SuHx group, lysis of myofibrils, degeneration of numerous mitochondria, vacuole formation, and dissociation of intercalated discs were observed in the cardiomyocytes. Intriguingly, degeneration of capillaries was 


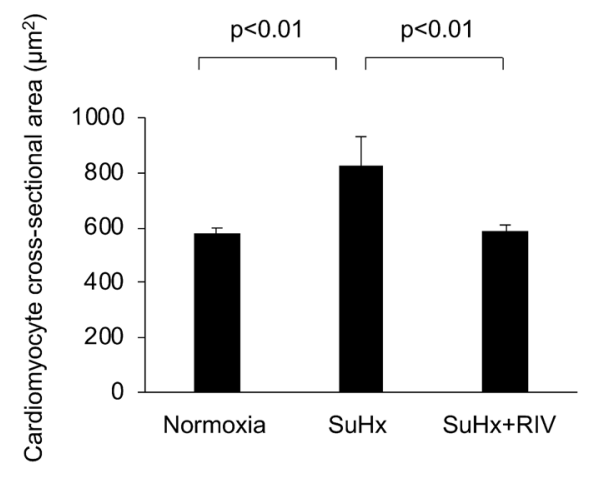

C

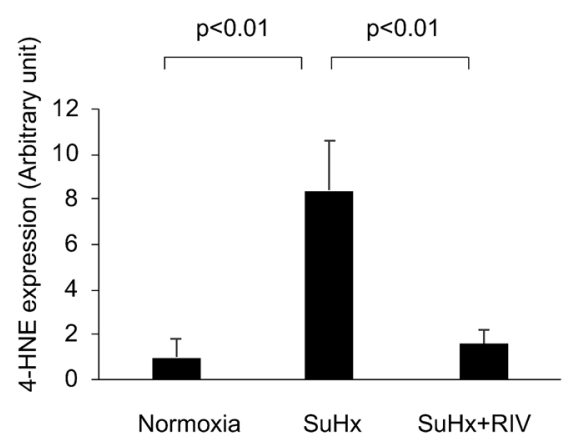

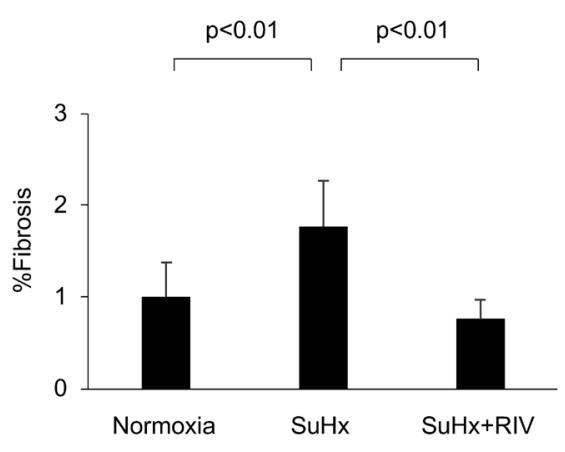

d

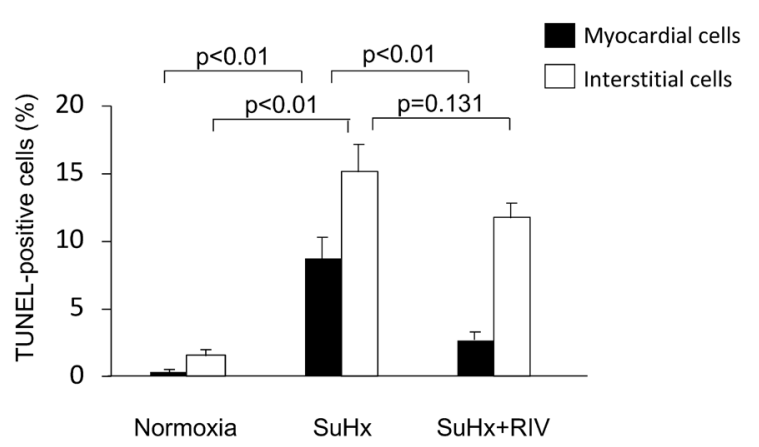

Fig. 3. Quantitative Measurement of Cardiomyocyte Cross-Sectional Area, Interstitial Fibrosis, 4-HNE-Modified Protein Adducts, and TUNELPositive Myocardial and Interstitial Cells from Photomicrographs Using ImageJ Software

Subcutaneous injection of Sugen5416 and exposure to hypoxia (SuHx) significantly increased the cross-sectional area of the cardiomyocyte (a), degree of interstitial fibrosis (\%fibrosis) (b), 4-HNE-modified protein adducts (c) and percentage of TUNEL-positive myocardial and interstitial cells (d) in the right ventricular myocardium. Rivaroxaban showed significant suppression of these changes (a, $N=3-4 ; \mathrm{b}, N=3 ; \mathrm{c}, N=3-5, \mathrm{~d}, N=3$ ). Data are shown as the mean and S.D.

prominent in the SuHx group, associated with thrombus formation in the capillary lumen (Figs. 4b-d). Treatment with rivaroxaban tended to prevent these ultrastructural changes (Figs. 4e, f).

4-HNE-Modified Protein Adducts Immunohistochemical staining revealed that 4-HNE-modified protein adducts in the RV myocardium in the SuHx group were significantly increased compared with the Normoxia group and that on the other hand, the increase was significantly reduced by administration of rivaroxaban (Figs. $2 \mathrm{~g}-\mathrm{i}, 3 \mathrm{c}$ ).

TUNEL Assay Findings In the TUNEL assay, a significant increase in the percentages of TUNEL-positive myocardial and interstitial cells were seen in the SuHx group compared with the Normoxia group. However, when treated with rivaroxaban, the percentage of TUNEL-positive interstitial cells showed a decreasing trend while the percentage of TUNEL-positive myocardial cells was significantly suppressed (Figs. 2j-1, 3d).

Real-Time RT-PCR In the real-time RT-PCR assay, the expression of PAR-2 (Fig. 5a), ERK-1 (Fig. 5b), ERK-2 (Fig. $5 \mathrm{c}$ ), JNK (Fig. 5d), NF- $\kappa$ B (Fig. 5e), and TGF- $\beta$ (Fig. 5f) mRNA was significantly higher in the SuHx group compared to that in the Normoxia group. In contrast, the expression of these mRNAs was significantly lower in the SuHx + RIV group than in the SuHx group.

Western Blotting for Expression of Various Molecules by
HCMECs In HCMECs, the ratios of PAR-2 to $\beta$-actin (Fig. 6a), p-ERK-1/2 to ERK-1/2 (Fig. 6 b), p-NF- $\kappa$ B to NF- $\kappa$ B (Fig. 6c), p-JNK to JNK (Fig. 6d) and p-Smad3 to Smad3 (Fig. 6e) expression were significantly higher in the SuHx group than in the Normoxia group in the Western blotting assay. These ratios in the SuHx + RIV group were significantly lower than those in the SuHx group.

\section{DISCUSSION}

This study demonstrated that subcutaneous injection of Sugen 5416 followed by $14 \mathrm{~d}$ of exposure to hypoxia $(10 \pm 1 \%$ $\mathrm{O}_{2}$ ) caused interstitial fibrosis and cardiomyocyte hypertrophy in the RV myocardium of rats. It is known that patients and animals with PAH frequently develop RV remodeling and heart failure. ${ }^{16,17)}$ We found that exposing rats to hypoxia for $14 \mathrm{~d}$ after administration of Sugen5416 resulted in RV remodeling. However, the RV changes were attenuated in rats that were treated with rivaroxaban.

In the model rats, RV systolic pressure and Fulton index were significantly decreased when administered rivaroxaban. In addition, increased medial wall thickness with plexiform lesion in pulmonary arterioles was improved. These observations indicate that pulmonary artery systolic pressure and RV hypertrophy were attenuated by rivaroxaban. However, the degree of the decrease in RV systolic pressure and Fulton index 

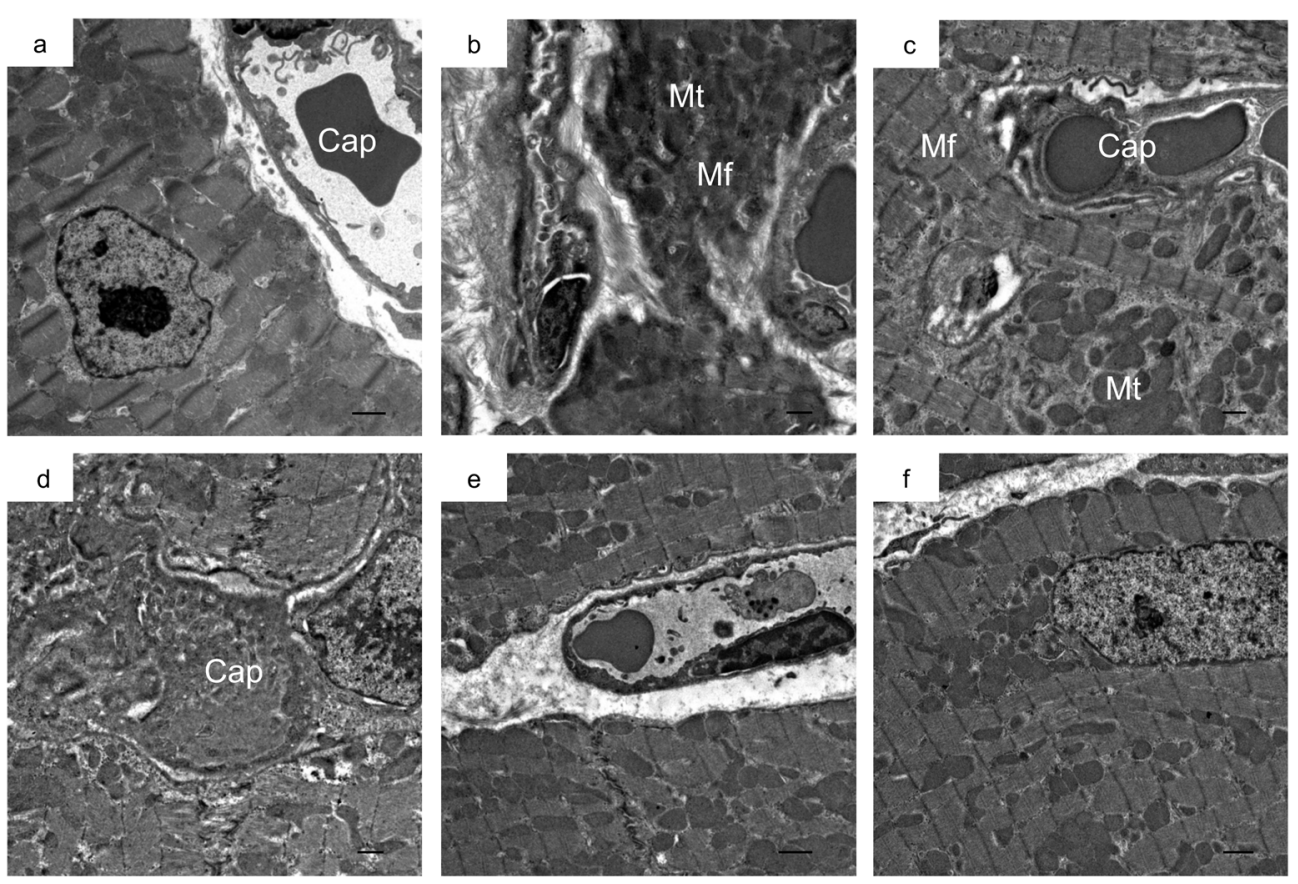

Scale bar $=1 \mu \mathrm{m}$.

Fig. 4. Representative Electron Micrographs of the Right Ventricular (RV) Myocardium in Rats

Myocardial cells kept under normoxia appear normal ultrastructural configuration (a). Rats exposed to hypoxia after injection of Sugen5416 (SuHx group) showed myofibrillar lysis, degeneration of mitochondria, and capillary occlusion (b-d). Treatment with rivaroxaban attenuated endothelial cell degeneration and preserved normal ultrastructural configuration (e, f). Cap, capillary; Mf, myofibers, Mt, mitochondria.

a

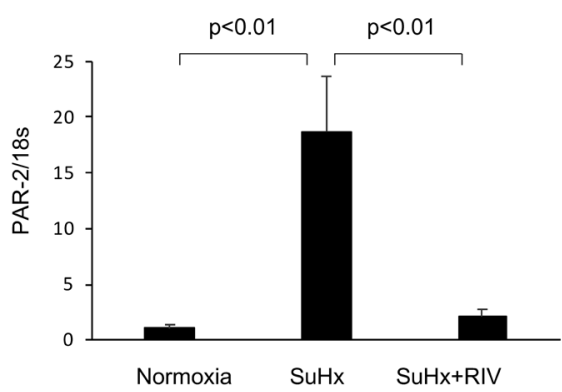

d

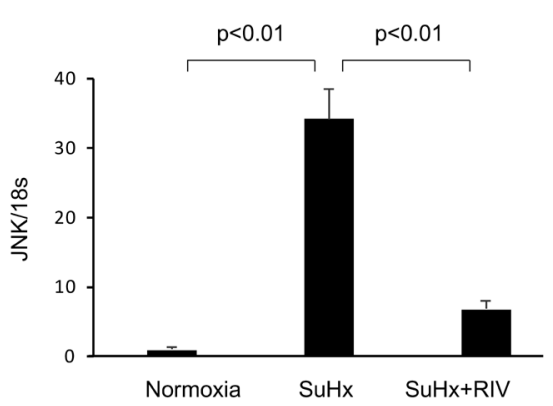

b

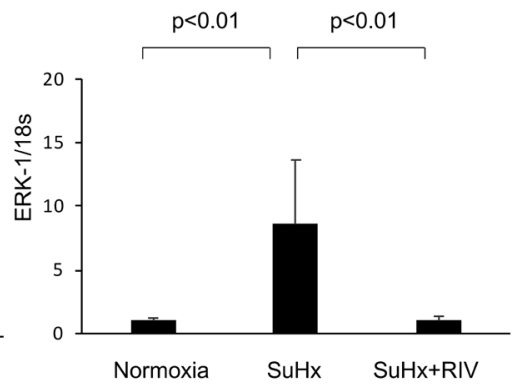

c

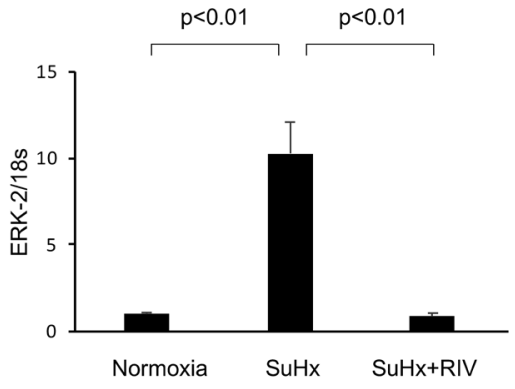

f

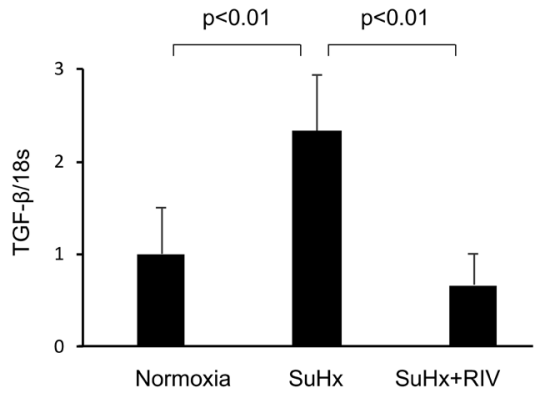

Fig. 5. Quantitative mRNA Analysis Using Real-Time RT-PCR in Right Ventricular (RV) Myocardial Tissue

In the RV myocardial tissue, expression of PAR-2 (a, $N=3-6)$, ERK-1 (b, $N=4-5)$, ERK-2 (c, $N=4-5)$, JNK (d, $N=3$ ), NF- $\kappa \mathrm{B}(\mathrm{e}, N=3-6)$, and TGF- $\beta$ (f, $N=3$ ) mRNAs was increased when administered Sugen5416 under hypoxic condition (SuHx). These mRNAs were significantly downregulated through treatment with rivaroxaban (RIV). The expression of each mRNA in the Normoxia group was not significantly different from that of the SuHx + RIV group. Data are shown as the mean and S.D.

was not sufficient to achieve normal levels. For the echocardiographic parameters, FAC and TAPSE, there were no differences that were found significant between the SuHx group and the SuHx + RIV group, although both parameters showed a trend toward improvement. On the other hand, a significant decrease in RV Tei index, which has been shown to be clini- 


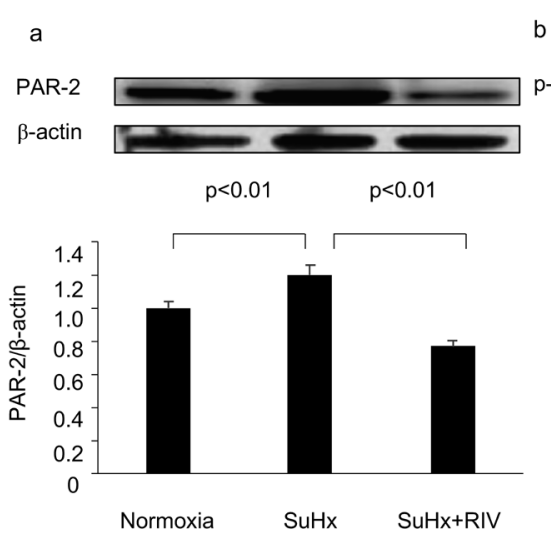

b
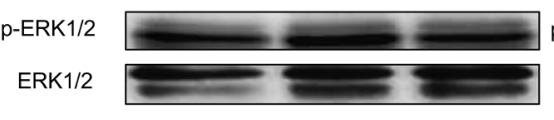

C
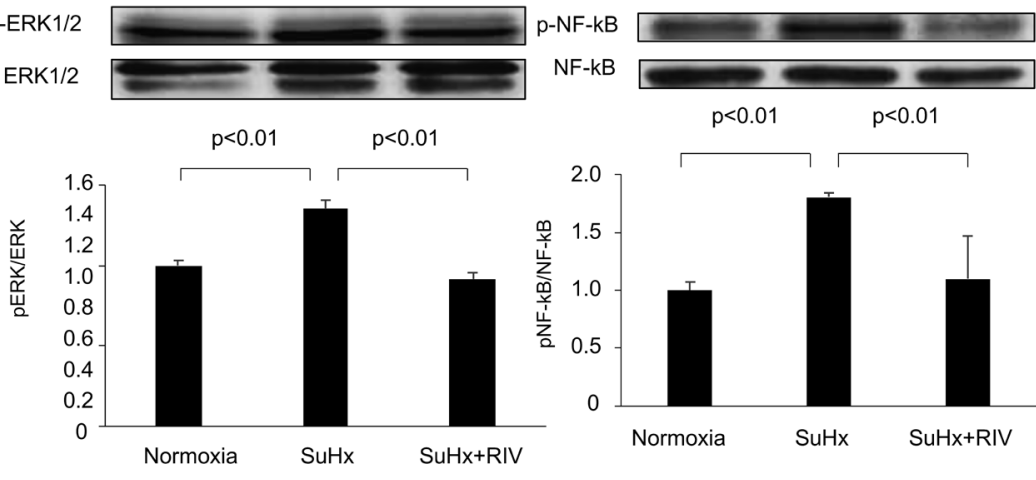

d

e
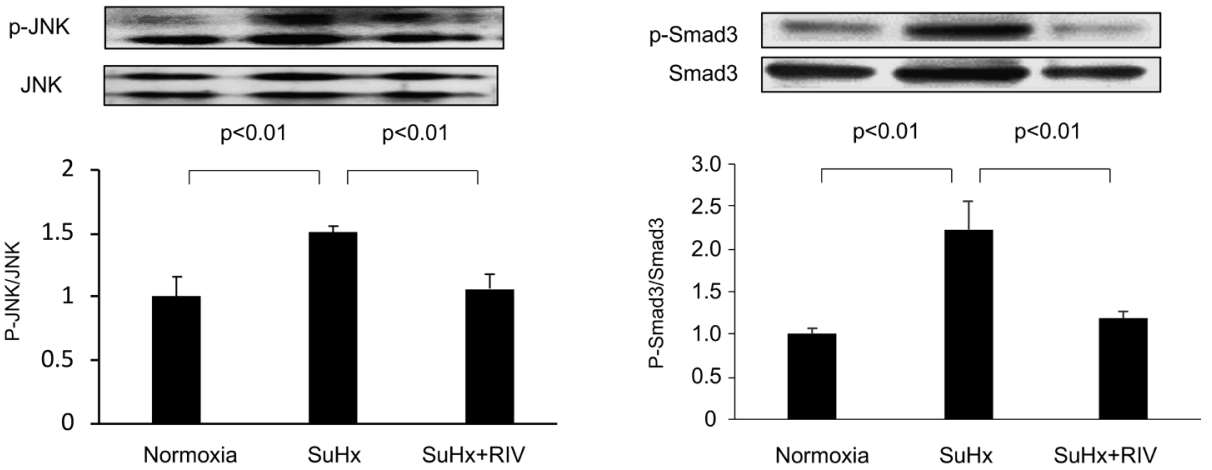

Fig. 6. Protein Expression Analysis by Western Blot in HCMECs

In HCMECs, the ratio of protein expression of PAR-2 to $\beta$-actin (a, $N=3$ ) was increased by treatment with Sugen5416 and exposure to hypoxia (SuHx). The ratios of phosphorylated proteins to unphosphorylated proteins in ERK-1/2 (b, $N=3$ ), NF- $\kappa \mathrm{B}(\mathrm{c}, N=3)$, JNK $(\mathrm{d}, N=3)$, and Smad3 (e, $N=3)$ were increased by treatment with Sugen 5416 and exposure to hypoxia (SuHx). PAR-2 expression was significantly downregulated by treatment with rivaroxaban (RIV). Phosphorylation of ERK-1/2, NF- $\kappa$ B, JNK, and Smad3 was significantly downregulated by treatment with RIV. Data are shown as the mean and S.D.

cally useful for predicting disease severity of PAH, was found in the SuHx + RIV group compared with the SuHX group.

Thrombus formation is known to have a role in the PAH pathogenesis. An increase in the expression of tissue factor (TF), the primary activator of the coagulation cascade that also has an essential role in hemostasis and thrombosis, has been observed in patients with $\mathrm{PAH}^{18,19)}$ as well as in mouse and rat models of PAH. ${ }^{20-22)}$ The complex of TF with factor VIIa (FVIIa) activates factor $\mathrm{X}$ to FXa. Hypoxia upregulates TF/ FVIIa activity and increases FXa activity. ${ }^{23,24)} \mathrm{FXa}$ activates PAR-2 in various cells. As expected, hypoxia with Sugen5416 increased the expression of PAR-2 protein in HCMECs.

In this study, the mechanism of rivaroxaban was examined in HCMECs because the amount of PAR-2 expression in endothelial cells has been reported to be larger than that of cardiomyocytes. ${ }^{25}$ In addition, there are multiple reports on the interaction between vascular endothelial cells and cardiomyocytes. ${ }^{26-28)}$ Therefore, it is considered that HCMECs may play an important role to attenuate the cardiomyocytes.

Administration of rivaroxaban to PAH model rats significantly downregulated the expression of PAR-2, ERK-1, JNK, and NF- $\kappa \mathrm{B}$ mRNAs. In addition, the elevated expression of PAR-2, p-ERK, p-JNK, and p-NF- $\kappa$ B proteins in HCMECs was significantly decreased by rivaroxaban administration. This indicates that rivaroxaban suppresses the activation of ERK, JNK, and NF- $\kappa \mathrm{B}$ pathways and the expression of PAR-2.

We demonstrated that 4-HNE-modified protein adducts increase in the RV myocardium of PAH model rats. From this observation, it was suggested that oxidative stress contribute in the development of RV dysfunction in the present rat model. These changes were suppressed when treated with rivaroxaban, suggesting that generation of reactive oxygen species (ROS) may have been reduced by rivaroxaban in our rat model of $\mathrm{PAH}$.

The NF- $\kappa$ B pathway is activated by ROS. Several studies have shown a relationship between this pathway and fibrosis. One study reported that activation of fibroblasts for the fibrotic process may be stimulated by FXa through the NF- $\kappa \mathrm{B}$ pathway, along with an elevation of ROS. ${ }^{29)}$ In our study, exposure of rats to hypoxia after Sugen5416 administration led to interstitial fibrosis, increased ROS production, and NF- $\kappa \mathrm{B}$ activation. However, these changes were significantly alleviated by administration of rivaroxaban. The increased expression of TGF- $\beta$ and activation of Smad3, a protein known to be strongly activated during fibrogenesis, were suppressed in hypoxia with Sugen5416 after rivaroxaban administration. These observations suggest that rivaroxaban suppresses fibrosis by inhibition of NF- $\kappa \mathrm{B}$ signaling.

FXa induces activation of ERK through signaling pathways that are initiated by PAR-2 activation. ${ }^{30)}$ Activation of PAR-2 leads to upregulation of the NF- $\kappa \mathrm{B}$ and ERK pathways. ${ }^{31)}$ Cardiac remodeling is attenuated by inhibition of NF- $\kappa \mathrm{B}$ signaling pathway in a rat model. ${ }^{32)}$ Our previous study revealed that expression of $\mathrm{p}-\mathrm{ERK}$ and $\mathrm{p}-\mathrm{NF}-\kappa \mathrm{B}$ in cardiomyocytes and vascular tissue was increased by hypoxia ${ }^{9)}$ and that ERK sig- 
naling pathway accelerates RV remodeling. ${ }^{4)}$ Treatment with rivaroxaban decreased ROS generation and fibrosis due to hypoxia with Sugen5416 in our PAH model rats. Accordingly, it can be suggested that activation of ERK and NF- $\kappa$ B causes $\mathrm{RV}$ remodeling, while rivaroxaban suppresses these pathways.

In addition, the activation of JNK protein in HCMECs was significantly decreased by rivaroxaban administration. FXa promotes JNK phosphorylation in human coronary artery endothelial cells. ${ }^{33)}$ Several studies have showed that PAR-2 activation enhances the phosphorylation of JNK depending on the cell type, such as in endothelial cells. ${ }^{34-37)}$ Moreover, the activation of JNK pathway as well as ERK pathway has been reported to promote cardiac hypertrophy. ${ }^{38,39)}$ The results of cardiomyocyte cross-sectional area and overall morphology of the cardiac section (see Supplementary Fig. 1, which shows representative light micrographs of overall morphology of the cardiac section) support the assumption that rivaroxaban attenuates cardiac hypertrophy. These findings indicate that rivaroxaban attenuate cardiac hypertrophy that causes RV remodeling, by suppressing the activation of JNK and ERK.

In the TUNEL assay, the effect of rivaroxaban was different between interstitial cells and myocardial cells. The number of TUNEL-positive cells was greater in interstitial cells compared with that in myocardial cells, suggesting that the damage caused in the interstitial cells was larger than that in myocardial cells. It may be for this reason that the effect of rivaroxaban in interstitial cells was not significant.

We have reported that TUNEL-positive myocardial cells can be found in lean mice suffering from intermittent hypoxia-induced cardiac remodeling. ${ }^{15)}$ In this study, rivaroxaban was found to decrease TUNEL-positive cells. In addition, it has been reported that the activation of $\mathrm{ERK}^{40)}$ and $\mathrm{JNK}^{41)}$ leads to apoptosis. In our study, we found that rivaroxaban suppressed the SuHx-induced activation of ERK and JNK. It is suggested from these observations that the prevention of cardiac cell death by rivaroxaban, may be one factor that contribute to the attenuation of SuHx-induced cardiac remodeling.

The present findings suggested that rivaroxaban could improve RV remodeling in PAH model rats through the suppression of the combined effect produced from multiple signaling pathways, including ERK, JNK, and NF- $\kappa \mathrm{B}$, associated with PAR-2. Capillary degeneration and thrombotic occlusion of vessels were observed in the RV myocardium of the SuHx group, while these changes were attenuated by rivaroxaban administration. Excessive activation of coagulation and inadequate anticoagulation might be involved in the pathophysiology of some types of RV remodeling caused by pulmonary hypertension, including chronic thromboembolic pulmonary hypertension (CTEF). ${ }^{42}$ Thus, although treatment with rivaroxaban alone is not sufficient for a complete recovery of $\mathrm{PAH}$, rivaroxaban is considered to have beneficial effects on improving the pathological changes of PAH.

One limitation in our study is that although we showed that the ratios of PAR-2 to $\beta$-actin, $\mathrm{p}$-ERK-1/2 to ERK-1/2, $\mathrm{p}-\mathrm{NF}-\kappa \mathrm{B}$ to NF- $\kappa \mathrm{B}, \mathrm{p}-\mathrm{JNK}$ to JNK, and $\mathrm{p}-\mathrm{Smad} 3$ to $\mathrm{Smad} 3$ were suppressed by rivaroxaban in HCMECs, these results have not been validated in the RV myocardium of PAH model rats.

In conclusion, treatment with rivaroxaban attenuated $\mathrm{RV}$ remodeling in PAH model rats by reducing ERK, JNK, and $\mathrm{NF}-\kappa \mathrm{B}$ activation. Rivaroxaban has the possibility of provid- ing additive effects on RV remodeling in patients with PAH.

Acknowledgments This research was supported by JSPS Kakenhi Grant (No. 23590267) from the Osaka University of Pharmaceutical Sciences, and also by research funding from Bayer Yakuhin, Ltd. under a contract for investigator-initiated research. The funders had no role in study design, data collection, data analysis, or preparation of the manuscript.

We are grateful to C. Ohta, Y. Ogami, and Y. Fujioka for their expert technical assistance. We also acknowledge enthusiastic support from S. Matsumoto, A. Hosokawa, G. Takechi, H. Wada, S. Kondo, A. Tanihata, T. Ogata, and K. Kanesaki (research assistants at the Laboratory of Cardiovascular Pharmacotherapy and Toxicology, Osaka University of Pharmaceutical Sciences).

Conflict of Interest Dr. T. Hayashi received research funding from Bayer Yakuhin, Ltd. The other authors declare no conflict of interest.

Supplementary Materials The online version of this article contains supplementary materials.

\section{REFERENCES}

1) Yamashita $\mathrm{C}$, Hayashi $\mathrm{T}$, Mori $\mathrm{T}$, Tazawa N, Kwak CJ, Nakano $\mathrm{D}$ Sohmiya K, Okada Y, Kitaura Y, Matsumura Y. Angiotensin II receptor blocker reduces oxidative stress and attenuates hypoxiainduced left ventricular remodeling in apolipoprotein E-knockout mice. Hypertens. Res., 30, 1219-1230 (2007).

2) Abe K, Toba M, Alzoubi A, Ito M, Fagan KA, Cool CD, Voelkel NF, McMurtry IF, Oka M. Formation of plexiform lesions in experimental severe pulmonary arterial hypertension. Circulation, 121, $2747-2754$ (2010)

3) Litz J, Sakuntala Warshamana-Greene G, Sulanke G, Lipson KE, Krystal GW. The multi-targeted kinase inhibitor SU5416 inhibits small cell lung cancer growth and angiogenesis, in part by blocking Kit-mediated VEGF expression. Lung Cancer, 46, 283-291 (2004).

4) Woo E, Kato R, Imano H, Fujiwara Y, Ijiri Y, Okada Y, Yamaguchi T, Izumi Y, Yoshiyama M, Katsumata T, Hayashi T. Capillary degeneration and right ventricular remodeling due to hypoxic stress with Sugen5416. Curr. Vasc. Pharmacol., 15, 589-598 (2017).

5) Badesch DB, Abman SH, Simonneau G, Rubin LJ, McLaughlin VV. Medical therapy for pulmonary arterial hypertension: updated ACCP evidence-based clinical practice guidelines. Chest, 131, 1917-1928 (2007).

6) Perzborn E, Roehrig S, Straub A, Kubitza D, Mueck W, Laux V. Rivaroxaban: a new oral factor Xa inhibitor. Arterioscler. Thromb. Vasc. Biol., 30, 376-381 (2010).

7) Delbeck M, Nickel KF, Perzborn E, Ellinghaus P, Strassburger J, Kast R, Laux V, Schäfer S, Schermuly RT, von Degenfeld G. A role for coagulation factor $\mathrm{Xa}$ in experimental pulmonary arterial hypertension. Cardiovasc. Res., 92, 159-168 (2011).

8) Kwapiszewska G, Markart P, Dahal BK, Kojonazarov B, Marsh LM, Schermuly RT, Taube C, Meinhardt A, Ghofrani HA, Steinhoff M, Seeger W, Preissner KT, Olschewski A, Weissmann N, Wygrecka M. PAR-2 inhibition reverses experimental pulmonary hypertension. Circ. Res., 110, 1179-1191 (2012).

9) Imano H, Kato R, Tanikawa S, Yoshimura F, Nomura A, Ijiri Y, Yamaguchi T, Izumi Y, Yoshiyama M, Hayashi T. Factor Xa inhibition by rivaroxaban attenuates cardiac remodeling due to intermittent hypoxia. J. Pharmacol. Sci., 137, 274-282 (2018).

10) Di Bello V, Conte L, Delle Donne MG, Giannini C, Barletta V, Fabiani I, Palagi C, Nardi C, Dini FL, Marconi L, Paggiaro P, Palla 
A, Marzilli M. Advantages of real time three-dimensional echocardiography in the assessment of right ventricular volumes and function in patients with pulmonary hypertension compared with conventional two-dimensional echocardiography. Echocardiography, 30, 820-828 (2013).

11) Kusumoto A, Miyata M, Kubozono T, Ikeda Y, Shinsato T, Kuwahata S, Fujita S, Takasaki K, Yuasa T, Hamasaki S, Tei C. Highly sensitive cardiac troponin $\mathrm{T}$ in heart failure: comparison with echocardiographic parameters and natriuretic peptides. J. Cardiol., 59, 202-208 (2012).

12) Sahin M, Alizade E, Pala S, Alici G, Ozkan B, Akgun T, Emiroglu Y, Demir S, Yazicioglu MV, Turkmen MM. The effect of cilostazol on right heart function and pulmonary pressure. Cardiovasc. Ther., 31, e88-e93 (2013)

13) Duran JM, Makarewich CA, Trappanese D, Gross P, Husain S, Dunn J, Lal H, Sharp TE, Starosta T, Vagnozzi RJ, Berretta RM, Barbe M, Yu D, Gao E, Kubo H, Force T, Houser SR. Sorafenib cardiotoxicity increases mortality after myocardial infarction. Circ. Res., 114, 1700-1712 (2014).

14) Hayashi $T$, James $T N$, Buckingham DC. Ultrastructure and immunohistochemistry of the coronary chemoreceptor in human and canine hearts. Am. Heart J., 129, 946-959 (1995).

15) Inamoto $S$, Yoshioka $T$, Yamashita $C$, Miyamura $M$, Mori $T$, Ukimura A, Matsumoto C, Matsumura Y, Kitaura Y, Hayashi T. Pitavastatin reduces oxidative stress and attenuates intermittent hypoxia-induced left ventricular remodeling in lean mice. Hypertens. Res., 33, 579-586 (2010).

16) van der Bruggen CEE, Tedford RJ, Handoko ML, van der Velden J, de Man FS. RV pressure overload: from hypertrophy to failure. Cardiovasc. Res., 113, 1423-1432 (2017).

17) Daicho $T$, Yagi $T$, Takano S, Marunouchi $T$, Abe $Y$, Ohara M, Takeo S, Tanonaka K. Alterations in pharmacological action of the right ventricle of monocrotaline-induced pulmonary hypertensive rats. Biol. Pharm. Bull., 32, 1378-1384 (2009).

18) Berger G, Azzam ZS, Hoffman R, Yigla M. Coagulation and anticoagulation in pulmonary arterial hypertension. Isr. Med. Assoc. J., 11, 376-379 (2009).

19) Altman R, Scazziota A, Rouvier J, Gurfinkel E, Favaloro R, Perrone S, Fareed J. Coagulation and fibrinolytic parameters in patients with pulmonary hypertension. Clin. Cardiol., 19, 549-554 (1996).

20) White RJ, Meoli DF, Swarthout RF, Kallop DY, Galaria II, Harvey JL, Miller CM, Blaxall BC, Hall CM, Pierce RA, Cool CD, Taubman MB. Plexiform-like lesions and increased tissue factor expression in a rat model of severe pulmonary arterial hypertension. $\mathrm{Am}$. J. Physiol. Lung Cell. Mol. Physiol., 293, L583-L590 (2007).

21) White RJ, Galaria II, Harvey J, Blaxall BC, Cool CD, Taubman MB. Tissue factor is induced in a rodent model of severe pulmonary hypertension characterized by neointimal lesions typical of human disease. Chest, 128 (Suppl.), 612S-613S (2005).

22) Bauer EM, Zheng H, Comhair S, Erzurum S, Billiar TR, Bauer PM. Complement $\mathrm{C} 3$ deficiency attenuates chronic hypoxia-induced pulmonary hypertension in mice. PLoS ONE, 6, e28578 (2011).

23) Monteiro RQ, Lima LG, Gonçalves NP, DE Souza MR, Leal AC, Demasi MA, Sogayar MC, Carneiro-Lobo TC. Hypoxia regulates the expression of tissue factor pathway signaling elements in a rat glioma model. Oncol. Lett., 12, 315-322 (2016).

24) Stavik B, Espada S, Cui XY, Iversen N, Holm S, Mowinkel MC, Halvorsen B, Skretting G, Sandset PM. EPAS1/HIF-2 alpha-mediated downregulation of tissue factor pathway inhibitor leads to a prothrombotic potential in endothelial cells. Biochim. Biophys. Acta, 1862, 670-678 (2016).

25) Cicala C. Protease activated receptor 2 and the cardiovascular sys- tem. Br. J. Pharmacol., 135, 14-20 (2002).

26) Talman V, Kivelä R. Cardiomyocyte-endothelial cell interactions in cardiac remodeling and regeneration. Front. Cardiovasc. Med., 5, 101 (2018).

27) Gemberling M, Karra R, Dickson AL, Poss KD. Nrg1 is an injuryinduced cardiomyocyte mitogen for the endogenous heart regeneration program in zebrafish. eLife, 4, e05871 (2015).

28) D'Uva G, Aharonov A, Lauriola M, Kain D, Yahalom-Ronen Y, Carvalho S, Weisinger K, Bassat E, Rajchman D, Yifa O, Lysenko M, Konfino T, Hegesh J, Brenner O, Neeman M, Yarden Y, Leor J, Sarig R, Harvey RP, Tzahor E. ERBB2 triggers mammalian heart regeneration by promoting cardiomyocyte dedifferentiation and proliferation. Nat. Cell Biol., 17, 627-638 (2015).

29) Kitasato L, Yamaoka-Tojo M, Hashikata T, Ishii S, Kameda R, Shimohama T, Tojo T, Ako J. Factor Xa in mouse fibroblasts may induce fibrosis more than thrombin. Int. Heart J., 55, 357-361 (2014).

30) Koo BH, Chung KH, Hwang KC, Kim DS. Factor Xa induces mitogenesis of coronary artery smooth muscle cell via activation of PAR-2. FEBS Lett., 523, 85-89 (2002).

31) Wang H, Moreau F, Hirota CL, MacNaughton WK. Proteinaseactivated receptors induce interleukin- 8 expression by intestinal epithelial cells through ERK/RSK90 activation and histone acetylation. FASEB J., 24, 1971-1980 (2010).

32) Han A, Lu Y, Zheng Q, Zhang J, Zhao Y, Zhao M, Cui X. Qiliqiangxin attenuates cardiac remodeling via inhibition of TGF- $\beta 1$ Smad3 and NF- $\kappa \mathrm{B}$ signaling pathways in a rat model of myocardial infarction. Cell. Physiol. Biochem., 45, 1797-1806 (2018).

33) Pham PT, Fukuda D, Yagi S, Kusunose K, Yamada H, Soeki T, Shimabukuro M, Sata M. Rivaroxaban, a specific FXa inhibitor, improved endothelium-dependent relaxation of aortic segments in diabetic mice. Sci Rep., 9, 11206 (2019).

34) Borensztajn K, Peppelenbosch MP, Spek CA. Factor Xa: at the crossroads between coagulation and signaling in physiology and disease. Trends Mol. Med., 14, 429-440 (2008).

35) Lidington EA, Steinberg R, Kinderlerer AR, Landis RC, Ohba M, Samarel A, Haskard DO, Mason JC. A role for proteinase-activated receptor 2 and PKC-epsilon in thrombin-mediated induction of decay-accelerating factor on human endothelial cells. Am. J. Physiol. Cell Physiol., 289, C1437-C1447 (2005).

36) Moriyuki K, Nagataki M, Sekiguchi F, Nishikawa H, Kawabata A. Signal transduction for formation/release of interleukin- 8 caused by a PAR2-activating peptide in human lung epithelial cells. Regul. Pept., 145, 42-48 (2008).

37) Zhang X, Wang W, Mize GJ, Takayama TK, True LD, Vessella RL. Protease-activated receptor 2 signaling upregulates angiogenic growth factors in renal cell carcinoma. Exp. Mol. Pathol., 94, 91-97 (2013).

38) $\overline{\mathrm{Hu} B}$, Song JT, Ji XF, Liu ZQ, Cong ML, Liu DX. Sodium ferulate protects against angiotensin II-induced cardiac hypertrophy in mice by regulating the MAPK/ERK and JNK pathways. Biomed Res Int., 2017, 3754942 (2017)

39) Xiao J, Jiang H, Zhang R, Fan G, Zhang Y, Jiang D, Li H. Augmented cardiac hypertrophy in response to pressure overload in mice lacking ELTD1. PLOS ONE, 7, e35779 (2012).

40) Cagnol S, Chambard JC. ERK and cell death: mechanisms of ERKinduced cell death - apoptosis, autophagy and senescence. FEBS J., 277, 2-21 (2010).

41) Dhanasekaran DN, Reddy EP. JNK signaling in apoptosis. Oncogene, 27, 6245-6251 (2008).

42) Lang IM, Dorfmüller P, Vonk Noordegraaf A. The pathobiology of chronic thromboembolic pulmonary hypertension. Ann. Am. Thorac. Soc., 13 (Suppl. 3), S215-S221 (2016). 